\title{
Achtsamkeit in der Praxis der Traumatherapie
}

\section{Potential, Anwendungsmöglichkeiten und Grenzen von achtsamkeitsbasierten Interventionen}

\author{
Sabine Fabach (iD)
}

Eingegangen: 23. Januar 2017 / Angenommen: 10. Mai 2017 / Online publiziert: 29. Mai 2017

(c) Der/die Autor(en) 2017. Dieser Artikel ist eine Open-Access-Publikation.

Zusammenfassung Achtsamkeit findet zunehmend Beachtung und Verwendung im psychotherapeutischen Kontext. In diesem Artikel soll anhand der verschiedenen Aspekte von Achtsamkeit gezeigt werden, dass viele etablierte Methoden innerhalb der Traumatherapie bereits auf Prinzipien der Achtsamkeit basieren. Darauf aufbauend wird untersucht, wo individuell adaptierte Anwendung von Achtsamkeitsübungen in der Ressourcen- und Stabilisierungsphase noch gezielter eingesetzt werden können und wie die dabei trainierten Fähigkeiten die Wirksamkeit der Techniken der Konfrontationsphase verbessern oder erst anwendbar machen. Dabei stellt sich auch die Frage, inwieweit Eigenerfahrung mit Meditation für Therapeut_innen zu empfehlen ist, um Achtsamkeit hilfreich in die eigene Praxis integrieren zu können.

Schlüsselwörter Traumatherapie - Achtsamkeit · Affektregulation · Selbstwirksamkeit

Mindfulness in the practise of trauma therapy The potential, possible applications and limits of mindfulness based interventions

Summary Increasingly, mindfulness is both finding recognition as well as being utilized in the field of psychotherapy. Through an exploration of different aspects of mindfulness, this article seeks to demonstrate that many established methods within trauma therapy already function on the basis of mindfulness principles. Progressing from this base, questions are examined as to where specific adaptions of mind-

Diese Arbeit ist Teil des Leitthemas „Traumatherapie in der Praxis“.

\section{S. Fabach $(\bowtie)$}

Mittelgasse 4/3/14, 1060 Wien, Österreich

praxis@fabach.at fulness exercises can be applied with more purposefully in the phase of building resources and stabilizing. Also, how improving the mindfulness abilities of a client can make the techniques implemented in the confrontation phase of therapy possible (for the first time) or improve their effectiveness. Finally, the question arises to what extent a personal meditation practise is to be recommended for therapists who wish to integrate mindfulness in their therapeutic work.

Keywords Traumatherapy · Mindfulness · Affect regulation · Self-efficacy

\section{Potenziale, Anwendungsmöglichkeiten und Gren- zen von achtsamkeitsbasierten Interventionen}

Achtsamkeit hat in den letzten 20 Jahren auch im psychotherapeutischen Kontext Einzug gehalten. Studien, Artikel und Bücher zu diesem Thema versuchen die Beziehungen und den Nutzen dieses 2500 Jahre alten Konzepts zur modernen Psychotherapie sichtbar zu machen (Heidenreich und Michalak 2009). So weist Reddemann (2016) darauf hin, „dass es gewisse Formen von Achtsamkeit und Mitgefühl als impliziertes Prinzip in den meisten psychotherapeutischen Schulen schon immer gab und gibt" (S. 20).

Die humanistischen Therapieformen weisen durch die Betonung der bedingungslosen Wertschätzung und empathischen Präsenz viele Gemeinsamkeiten mit dem Konzept von Achtsamkeit auf. Des Weiteren definieren beide den unmittelbaren Kontakt mit den inneren Vorgängen und eine emphatische Beziehung zu diesen als heilsam (vgl. Renn 2011; BundschuhMüller 2009). 
Kognitiv-behavioral orientierte Therapeut_innen ${ }^{1}$ erkannten ihrerseits die kognitiven Möglichkeiten der achtsamkeitsbasierten Meditation, woraus sich die „dritte Welle in der Verhaltenstherapie“ entwickelte (Hayes et al. 2014; Heidenreich und Michalak 2009). Der Fokus lag auf dem bewussten Wahrnehmen aller mentalen Zustände, die auch positive und neutrale Kognitionen umfasste. Weiss und Harrer (2010) weisen in diesem Sinne darauf hin, dass die Wirksamkeit von Achtsamkeit nicht darin liegt, die Gedanken und Gefühle als solche zu verändern, sondern die Beziehung der Klient_innen zu ihren Gedanken und Gefühlen erfährt eine Veränderung, und Handlungsautomatismen werden durch Achtsamkeit unterbrochen.

In der tiefenpsychologisch fundierten Psychotherapie hat sich unter anderem Reddemann (2006, 2011a, 2011b) mit den Gemeinsamkeiten und Einsatzmöglichkeiten von achtsamkeitsbasierten Interventionen in der Psychotherapie und im Besonderen bei traumatischem Stress beschäftigt. Ihre Überlegungen zur Reduzierung dissoziativer Mechanismen durch das Stärken des gegenwärtigen Erlebens mithilfe von Achtsamkeit sollen hier ebenfalls berücksichtigt werden.

Auf den Überlegungen dieser verschiedenen Therapieströmungen basierend beschäftigt sich der vorliegende Artikel mit den Anwendungsmöglichkeiten von Achtsamkeit in der Praxis der Traumatherapie. Ein tiefes Verstehen von Achtsamkeit ist gerade bei Menschen mit komplexen Traumatisierungen wichtig, da bestimmte Formen von Achtsamkeitsinterventionen auch kontraindiziert sein können, was nachfolgend anhand der Handlungsmöglichkeiten in der Stabilisierungs- und Konfrontationsphase einer Traumatherapie sichtbar gemacht werden soll. Abschließend werden die Vorteile und die Notwendigkeit eines Achtsamkeitstrainings für Therapeut_innen beleuchtet. Damit soll ein Anstoß zu einer tieferen fachlichen Auseinandersetzung mit dem Thema Achtsamkeit in der Traumatherapie angeboten werden.

\section{Definition}

Kabat-Zinn (2003) beschreibt Achtsamkeit als ,jenes Gewahrsein, das entsteht, wenn sich die Aufmerksamkeit mit Absicht und ohne zu bewerten auf die Erfahrungen richtet, die sich von Moment zu Moment entfalten“ (S. 145; zit. u. übers. nach Harrer und Weiss 2016, S. 30; vgl. auch Germer 2009). Diese Erfahrung bezieht sich auf Empfindungen, Wahrnehmungen der Sinnesorgane, Gefühle und ihre körperliche Repräsentanz sowie Gedanken einschließlich Erinnerungen, Vorstellungen und andere mentale Inhalte (Grossman und Reddemann 2016). Zu bemerken, was jetzt gerade in mir geschieht, ermöglicht es auch, sich zu erinnern,

\footnotetext{
${ }^{1}$ In diesem Artikel wird im Sinne einer geschlechtergerechten Sprache der Unterstrich verwendet. Damit soll die Existenz von mehr als zwei Geschlechtern sprachlich sichtbar gemacht werden.
}

was heilsam ist (Wetzel 2011, S. 40; Bodhi 2011). Dies ist als ein Aspekt von Achtsamkeit gerade auch für die Traumatherapie sehr relevant, damit Klient_innen lernen können, aus schädlichen Handlungsmuster auszusteigen und förderliche Handlungen zu tun (Reddemann 2011b). Ein weitere relevanter Aspekt von Achtsamkeit findet sich in der „Haltung der sogenannten Absichtslosigkeit“ (Renn 2011, S. 92). Dies kann sich auf ein bestimmtes Gefühl, auf Gedanken oder Überzeugungen beziehen. Achtsames Gewahrsein ist ergebnisoffen, die Kraft liegt in der Bereitschaft wahrzunehmen, was da ist, ohne es sofort zu manipulieren oder zu bewerten. Das Gewahrsein des gegenwärtigen Moments meint aber nicht eine neutrale kühle Aufmerksamkeit, sondern ist immer mit Freundlichkeit und Mitgefühl verbunden. Zusammenfassend lässt sich Achtsamkeit definieren als ein Gewahrsein des eigenen, sich ständig verändernden Erlebens in einer nicht bewertenden, ergebnisoffenen und mitfühlenden Art und Weise. Dieses Gewahrsein ist verbunden mit der Erinnerung, was hilfreich ist und was nicht. Diese Definition liegt den weiteren Betrachtungen der Einsatzmöglichkeiten und Einschränkungen von Achtsamkeit zugrunde.

\section{Forschung}

Die Wirksamkeit von Achtsamkeit durch spezielle Trainings wie Mindfulness-Based Stress Reduktion, entwickelt (MBSR; Kabat-Zinn 2003) ist durch verschiedene Studien, u. a. für verschiedene Störungsbilder wie etwa Stress oder die Rückfallprävention von Depressionen, für Persönlichkeitsstörungen, Ängste und Zwänge bestätigt worden. So weist das Ergebnis einer Metaanalyse von Grossman et al. (2009) darauf hin, dass das MBSR-Training Menschen helfen kann, ihre klinischen Probleme zu bewältigen (vgl. auch Huppertz et al. 2013). Dieses Ergebnis wird unterstützt von Studien zu strukturellen Veränderungen im Gehirn aufgrund von Meditationstraining (Siegel 2007; Weiss und Harrer 2010). Interessant sind hier auch Studien, die sich speziell mit den Veränderungen bei traumabedingten Störungen beschäftigen: King et al. (2013) stellten fest, dass der signifikanteste Bereich der Verbesserung in der Reduzierung des Vermeidungsverhaltens $\mathrm{zu}$ finden ist (vgl. auch Banks et al. 2015). Des Weiteren zeigte sich in dieser Studie eine Verbesserung der allgemeinen Symptome der Posttraumatischen Belastungsstörung (PTBS), des subjektiven Schmerzempfindens sowie eine Reduzierung im Faktor Selbstbeschuldigungen. Die Studie von Stephenson et al. (2016) weisen darauf hin, dass sich neben reduzierten PTBS-Symptomen vor allem die Fähigkeit zum bewussten Handeln und zur NonReaktivität erhöhte. Es zeigte sich auch eine Verminderung beim Auftreten von Hyperarousal und bei emotionaler Abstumpfung. Valdez et al. (2016) belegten, dass die nichtbewertende freundliche Haltung im Achtsamkeitstraining ein wichtiger Faktor ist. Eine 
neutrale Haltung zum eigenen Erleben erwies sich als weniger wirksam, besonders bei beziehungstraumatisierten Menschen.

Die genannten Studien weisen in die Richtung, dass Achtsamkeitstraining die Reduktion von Vermeidungsverhalten und Stresserleben unterstützt und das Gefühl von Selbstwirksamkeit erhöht. In den nächsten Schritten wird dargestellt, inwieweit diese Qualitäten von Achtsamkeit bereits in die Praxis der Traumatherapie integriert sind.

\section{Achtsamkeit in der Traumatherapie}

In Traumatherapie begeben sich Klient_innen, die aufgrund bestimmter Erfahrungen immer wieder von Gefühlen, Bildern und Körpererinnerungen überflutet werden und daher ein oft komplexes System an Vermeidung und Dissoziation aufgebaut haben. Das Vertrauen in die Welt und in sich selbst ist massiv verunsichert. Die Wahrnehmung und das Erleben der Gegenwart werden durch die traumatischen Erfahrungen und die daraus entstandenen Konzepte über sich und die Welt überlagert. Der chronisch erhöhte Stresslevel und andere unkontrollierbare Körpersensationen können erschöpfend und beängstigend sein. Daher haben sich in der Traumatherapie Methoden etabliert, die je nach Traumatyp, Ich-Stärke und PTBSSymptomen darauf ausgerichtet sind, innere Stabilität und Ressourcen zu stärken und Fähigkeiten zu trainieren, die mehr Affektregulation ermöglichen und dadurch eine Traumaverarbeitung erlauben.

Achtsamkeitsübungen trainieren Fähigkeiten, die für die Traumatherapie durchaus relevant sind. Dies sind der Umgang mit Gefühlen, die Verankerung in der Gegenwart, die Selbstberuhigung und ein wertschätzender freundlicher Umgang mit sich selbst. Daher verwenden viele Therapeut_innen bereits Methoden, die das Potenzial von Achtsamkeit in einem gut kontrollierbaren Rahmen nützen. Reddemann (2011a) empfiehlt beispielsweise eine Kombination aus selbstberuhigenden Elementen und Elementen des Erkennens von inneren Prozessen, vor allem, wenn wenig innere Stabilität vorhanden ist. Auch Huber (2005) entwickelte unterschiedliche Achtsamkeitsübungen, die auf die körperliche Spannung oder Entspannung und das Kommen und Gehen von Gedanken, Gefühlen und Körperempfindungen aufmerksam machen.

Empfehlungen zu traditionellen Achtsamkeitspraktiken wie Meditation sind sehr genau zu prüfen, da sie im schlechtesten Fall sogar kontraindiziert sein können. Bei komplextraumatisierten und dissoziativen Klient_innen kann Meditation - die auf einem offenen ruhigen Verweilen beruht bei allem, was auftaucht, - zu einer Überflutung durch Emotionen und $\mathrm{zu}$ starken Dissoziationen führen (Reddemann 2006). Wenn Achtsamkeit zu therapeutischen Zwecken genutzt werden soll, muss daher das Augenmerk besonders auf die Ich-Stärkung gerichtet sein. Jede
Intervention soll traumaadaptiert sein und die Klient_innen nicht zusätzlich belasten. Es ist immer zu bedenken, dass manche Klient_innen bereit sind, Dinge zu tun, um den Therapeut_innen zu gefallen und dabei in Kauf zu nehmen, ihre eigenen Grenzen zu überschreiten. Daher ist es enorm wichtig, Achtsamkeit vorsichtig und sehr hellhörig anzuleiten. Generell gilt, dass Achtsamkeitsübungen das Erwachsenen-Ich stärken sollen, und je stärker dieses ist, umso mehr kann man achtsamkeitsbasierte Interventionen auch für die traumabelasteten Bereiche anbieten.

Im nächsten Abschnitt werden einige Interventionen und Teilziele der Stabilisierungsphase dahingehend untersucht, inwieweit diese der Definition von Achtsamkeit entsprechen und wo achtsame Interventionen noch stärker eingesetzt werden könnten.

\section{Achtsamkeit in der Stabilisierungsphase}

Die Stabilisierungsphase ist ein zentraler Bestandteil jeder Traumatherapie. Je komplexer, je länger und je früher sich die Traumatisierungen lebenszeitlich ereignet haben, umso länger und wichtiger ist diese Phase, bevor mit Traumakonfrontation begonnen werden kann. Generell geht es hier um die Stärkung oder Etablierung eines Erwachsenen-Ichs, die Fähigkeit, sich selbst zu beruhigen und positive Ressourcen auszubauen und nutzbar zu machen (vgl. Purtscher und Sack 2016).

Ein zentraler Aspekt von Achtsamkeit ist die Fähigkeit, die Aufmerksamkeit auf etwas Bestimmtes $\mathrm{zu}$ fokussieren und diese Fokussierung auch aktiv steuern zu können. Das bedarf innerer Beobachter_innen, die bemerken, wo unsere Aufmerksamkeit gerade verweilt und was dort zu bemerken ist. Diese innere Instanz macht es erst möglich, innere Prozesse zu beschreiben und den inneren Strudel von Emotion und Reaktion $\mathrm{zu}$ verlangsamen und $\mathrm{zu}$ beeinflussen (Weiss und Harrer 2010). Diese inneren Beobachter_innen sind immer auch mit einer Aktivierung im präfrontalen Cortex verbunden (Roediger 2011) und stärken somit das erwachsene gegenwärtige Denkvermögen.

Zuerst gilt es, die Entwicklung der inneren Beobachter_innen im Bereich positiver Erfahrungen und Gefühle zu unterstützen (vgl. Reddemann 2011b). Hierbei werden die Klient_innen eingeladen zu erforschen, welche angenehmen Erfahrungen mit dem eigenen Körper gemacht werden können. Was lösen Hören, Spüren, Schmecken in den Klient_innen aus, wie drückt sich Freude und Entspannung in ihrem Körper aus? Diese Achtsamkeitsübungen lehren das Erkennen der vorhandenen Fähigkeit, Freude empfinden zu können, ohne das Negative zu verdrängen oder zu ignorieren. Gerade bei komplex Traumatisierten ist bei der Wahrnehmung des Körpers immer die Möglichkeit in Betracht zu ziehen, dass sie dadurch getriggert werden oder große Teile ihres Körpers gar nicht wahrnehmen können. Hier braucht es ein offenes, neugieriges und vor allem gemeinsames Suchen 
nach neutralen oder positiven Körperstellen, auf die der Fokus gelenkt werden kann. Eine andere Möglichkeit bietet die Übung, die Klient_innen den Kontakt des Körpers mit dem Boden oder dem Stuhl spüren zu lassen. Das unterstützt sie darin, sich stabiler zu fühlen, das Hier und Jetzt besser wahrzunehmen, und dies wirkt beruhigend. Diese Erfahrung können sie in den Alltag mitnehmen und wiederholen.

Die nicht bewertende und offene Haltung haben die Therapeut_innen vorzuleben, ohne die meist ambivalenten Gefühle der Klient_innen dem eigenen Körper gegenüber $\mathrm{zu}$ ignorieren, sondern sie in die Achtsamkeitsübungen zu integrieren (vgl. Ebner und Rost 2008; Weiss und Harrer 2010).

Bei genügend Sicherheit kann die Achtsamkeit auf den Atem gelenkt werden, sie hat eine stärker beruhigende Wirkung. Reddemann (2011a) empfiehlt beispielsweise, dass es hier vorerst genügt, die Atembewegungen des Körpers wahrzunehmen. Die Klient_innen können dazu die Hand auf den Bauch oder Brustkorb legen und so die Atembewegungen spüren. Als zusätzliche Konzentrationshilfe können die Atemzüge gezählt werden. Selbstberuhigung ist ein wichtiges Ergebnis achtsamkeitsbasierter Übungen. Dabei müssen sich die Klient_innen immer sicher fühlen und die Gewissheit haben, diesen Prozess selbst kontrollieren zu können. Dazu wiederum braucht es die inneren Beobachter_innen, die in der Lage sind zu bemerken, ob durch die Übung körperliche Entspannung einsetzt oder negative Empfindungen wie Angst wieder stärker werden.

Als eine weitere Ressourcenübung hat sich die achtsame Wahrnehmung über die Sinnesorgane etabliert, z. B. auf Objekte mit bestimmten Farben, auf Geräusche, Gerüche oder sensorische Empfindungen $\mathrm{zu}$ achten und diese mit Wörtern $\mathrm{zu}$ beschreiben. Wahrzunehmen, was im Hier und Jetzt ist, entspricht genau der Definition von Achtsamkeitstraining.

Eine weitere Stärke der oben beschriebenen inneren Beobachter_innen ist deren Unterstützung, sich nicht mit allen Empfindungen zu identifizieren. Diese Haltung gegenüber Gefühlen und Körpersensationen ist ein wichtiger Teil jeder Achtsamkeitspraxis und enorm hilfreich im Umgang mit Flashbacks und EgoStates. Hier werden die Klient_innen eingeladen, ihre Gefühle aus der Ferne zu betrachten, Distanz zu halten und sie zu beschreiben. Damit machen sie die Erfahrung, dass sie schwierige Gefühle aus der Distanz ertragen lernen können und dabei handlungsfähig bleiben. Sie lernen, dass sie mehr sind als ein sie ganz überflutendes unangenehmen Gefühl (Reddemann 2011a). Dadurch eröffnet sich eine Wahlmöglichkeit, wie sie darauf reagieren möchten. So können die Klient_innen langsam beginnen, basierend auf dem Wissen, dass sie zu freudvoller Erfahrung fähig sind, bewusst zwischen schwierigen und positiven Empfindungen hin und her zu pendeln. Diese Kompetenz, eine hilfreiche Distanz zu den Gefühlen zu halten, ist auch für die Traumakonfrontation von zentraler Bedeutung.

Ein weiteres wichtiges Merkmal von Achtsamkeit ist die Fähigkeit, mit der Aufmerksamkeit immer wieder in den gegenwärtigen Moment zurückzukehren. Menschen mit PTBS werden oft von Erinnerungen oder Zukunftsängsten überflutet. Flashbacks und dissoziative Symptome lassen sie den Bezug zur Gegenwart verlieren. Durch das Üben, immer wieder die gegenwärtigen Empfindungen aus der Perspektive der inneren Beobachter_innen wahrzunehmen und von den inneren Bewertungen und Interpretationen $\mathrm{zu}$ trennen, lernen Klient_innen, sich in der Gegenwart zu verankern, auch wenn starke Gefühle in ihnen toben. Dies wird möglich, da Achtsamkeitsübungen die inneren Prozesse verlangsamen. Durch diese stärkere Gegenwärtigkeit können Klient_innen zunehmend lernen, die alten oft destruktiven Schutzreaktionen in den Anfängen zu erkennen und in Richtung heilsamerer Reaktionen zu beeinflussen. Es ist sehr herausfordernd, die eigene Innenwelt immer deutlicher wahrzunehmen, die Klient_innen sind dabei oft mit Scham und ihrer eigenen Verachtung konfrontiert. Daher ist es Aufgabe der Therapeut_innen, darauf zu achten, dass die Schritte verdaubar klein sind (vgl. Reddemann 2011a, S. 107).

Ein Teil der Wirksamkeit all dieser Übungen hängt auch von der inneren Haltung ab, die die Klient_innen zu sich haben. Offenheit, Freundlichkeit und nicht bewertendes Anerkennen aller Empfindungen und Gefühle beschreiben diese Grundhaltung, die es mit einzuüben gilt. Viele traumatisierte Klient_innen sind in ihrem Selbstwert, ihrem Vertrauen $\mathrm{zu}$ sich und der Welt erschüttert, Mitgefühl oder Freundlichkeit sich selbst gegenüber zu empfinden, ist kaum möglich. Es ist schon ein Erfolg, wenn die Klient_innen „zähneknirschend“ einem Gefühl der Verletzlichkeit oder einem inneren Kindanteil erlauben können, kurz da zu sein. Mitgefühl mit sich selbst zu entwickeln, für die verletzten wie auch für die wütenden Anteile, ist für viele traumatisierte Klient_innen ein sehr schwieriges Unterfangen. Daher ist bei jeder Achtsamkeitsübung darauf zu achten, dass Freundlichkeit und Selbstmitgefühl mit eingeübt wird. Freundliche Körpergesten können hier unterstützen. Hilfreich ist für viele, die Hand auf die schmerzende Körperstelle oder die Stelle zu legen, wo das schmerzliche Gefühl am direktesten zu spüren ist, und die Wärme der Hand auf das Gefühl zu visualisieren. Für einige fühlt sich diese Geste aber nicht richtig an. Als Alternative können sich die Klient_innen z. B. selbst am Handgelenk halten. Diese Gesten des Trostes erhöhen wieder das Gefühl der Selbstwirksamkeit im Sinne „Ich kann etwas für mich tun“.

Gleichzeitig können die Klient_innen spüren, dass ihr Mitgefühl mit sich selbst ihr Leiden mindert (Grossman und Reddemann 2016, S. 227). Diese Erfahrung, dass es wohltuend sein kann, einem schmerzlichen Gefühl oder inneren Anteil mitfüh- 
lend $\mathrm{zu}$ begegnen und in einer freundlichen Aufmerksamkeit zu halten, macht Klient_innen viel Mut. Aufbauend auf die geübten Fähigkeiten können mit der Zeit auch schwierigere Gefühle in dieser kontrollierten und heilsamen Form gespürt und damit verdaut werden.

$\mathrm{Zu}$ jeder Achtsamkeitsübung gehören auch das Loslassen und das Anerkennen, das die Gefühle sich ständig verändern. Zum Abschluss jeder achtsamkeitsbasierten Übung empfiehlt es sich daher, mit den Klient_ innen einen Schritt zum bewussten (Los-)Lassen der Empfindung zu finden. Dies kann passieren, indem die Klient_innen die Veränderung der Empfindung bemerken oder indem sie z. B. dem Gefühl erlauben, an einem passenden inneren Ort zu bleiben. Manchmal kann dieses (Los-)Lassen auch durch das Verschieben des Fokus der Aufmerksamkeit auf eine vorher positiv/neutral etablierte Körperstelle ermöglicht werden oder durch Übungen, die das kognitive Denkvermögen ansprechen.

Die Achtsamkeitsübungen zum Ziele der Stabilisierung sollen nach Möglichkeit auch außerhalb der Therapiestunde geübt werden, dazu braucht es eine klar definierte Aufgabenstellung. Ein häufiges Problem besteht darin, dass diese häufig vergessen werden, daher ist es sinnvoll, dass die Klient_innen sie für sich selbst auf einem Blatt Papier notieren. Man kann mit Übungen beginnen, die mit dem Alltag der Klient_innen verknüpft sind, z. B. achtsames Abwaschen, achtsames Hören auf dem Weg zur Arbeit, Spüren des Körpers beim Atmen, während des Teetrinkens in der Pause oder Achtsamkeit für den Geschmack des Essens. Wichtig ist hier, dass die Klient_innen sich entscheiden, wo Achtsamkeit für sie am leichtesten möglich ist.

Indem sie durch das stetige Üben zum gegenwärtigen Erleben zurückkommen, können die Klient_innen eher erkennen, wann ihr innerer Autopilot sich einschaltet oder sie sich durch Dissoziation abtrennen. Je öfter sie dies bemerken, umso höher ist die Wahrscheinlichkeit, dass sie sich erinnern, welche Reaktion hier hilfreich ist und welche nicht. Sich an Hilfreiches zu erinnern, ist ein wichtiger Effekt von Achtsamkeitstraining und eine Voraussetzung dafür, früh eingelerntes Vermeidungsverhalten zu verändern (Rytz und Wiesmann 2016).

In den Interventionen der Phase der Stabilisierung und des Ressourcenaufbaus finden sich bereits alle Aspekte von Achtsamkeit integriert bzw. sind explizit bereits als Achtsamkeitsübungen tituliert. Diese erlauben den Klient_innen in kleinen Schritten, sich den gegenwärtigen und den als traumatisch gespeicherten Erinnerungen zu nähern und damit Vermeidungsverhalten zu reduzieren. Die Übungen stärken das Erwachsenen-Ich und fungieren als hilfreiche Tools zur Selbstberuhigung.

Es stellt sich die Frage, inwieweit die Effekte von Achtsamkeitsübungen auch in der Traumakonfrontation zu finden sind.

\section{Achtsamkeit in der Traumakonfrontation}

Das Heilsame in all diesen Methoden der Konfrontation - ob Screentechnik, Eye Movement Desensitization and Reprocessing (EMDR) oder auch bei Constant Installation of Present Orientation and Safety (CIPOS) und Affektkette (Huber 2011) - ist die Fähigkeit der Klient_innen, ihre Emotionen, Körpersensationen und sensorischen Erinnerungen bei gleichzeitiger Verankerung im Hier und Jetzt halten und anerkennen zu können (Münker-Kramer 2015). Eine sehr ähnliche Beschreibung, die auch an den Prozess einer EMDR-Sitzung erinnert, findet sich in der folgenden Definition von Achtsamkeitsübungen durch Briere (2014), für den Achtsamkeit darin besteht, „sich auf einen einzigen Prozess zu konzentrieren ..., aufmerksam beim gegenwärtigen Moment zu bleiben und Gefühle und Gedanken kommen und gehen zu lassen, ohne sie festzuhalten. $\mathrm{Zu}$ den Dingen, die man so aufsteigen und wegfallen lässt, gehören Wertungen und Urteile über sich selbst und innere Erfahrungen“ (S. 438). Diese Fähigkeit, das gegenwärtige Erleben in eben dieser besonderen Art von Aufmerksamkeit halten und auch loslassen zu können, ist ein zentraler Moment, damit traumatische Erfahrungen verarbeitet werden können.

Für diese Verarbeitung, vor allem bei Bindungstraumata, ist es zusätzlich wichtig, dass die Klient_innen ein gewisses Maß an Selbstmitgefühl für sich entwickeln können. Immer wieder zeigt sich, dass das Stocken im Prozess des Durcharbeitens durch eine innere Weigerung, Selbstmitgefühl zu empfinden, ausgelöst wird. Beim EMDR wird z. B. mit der Frage „Was würdest du $\mathrm{zu}$ dem verletzten Kind sagen, wenn du als Erwachsene in das Bild hineingehen könntest“ versucht, eine mitfühlende Instanz in den Prozess zu integrieren. So kann z. B. bei einem/einer Klientin ein positives Introjekt einer früheren Bezugsperson diese Rolle des Mitgefühls und der Fürsorge für das verletzte innere Kind übernommen haben.

Dieses Mitgefühl wird auch in der Bildschirmtechnik (Screen-Technik) eingeladen. Der Erzählfluss wird immer wieder gestoppt, sodass die Klient_innen ihrem Empfinden im Hier und Jetzt nachspüren können und sich erlauben, diese Gefühle ganz bewusst wahrzunehmen. Der Aspekt des Mitgefühls kann z. B. durch die Frage „Was empfindest du jetzt, wenn du dieses Kind so auf der Leinwand siehst?" eingeflochten werden.

An diesen Beispielen ist zu erkennen, dass die Erfahrungen mit achtsamkeitsbasierten Interventionen in der Stabilisierungsphase auch für die Konfrontationsphase enorm hilfreich sind. Den Bezugspunkt innerer Beobachter_innen halten und die Fähigkeit zwischen schwierigen und positiven Emotionen hin und her pendeln zu können, sind gute Voraussetzungen. Gemeinsam mit dem Vertrauen, dass Gefühle sich verändern können und durch Selbstmitgefühl beruhigen lassen, entstehen ein stabiler Boden und gute innere 
Fertigkeiten für die Konfrontation und Verarbeitung traumatischer Erfahrungen.

\section{Achtsamkeitstraining für Traumatherapeut_innen}

Um die Klient_innen $\mathrm{zu}$ unterstützen, achtsam und mitfühlend mit sich selbst zu sein, ist es sicher hilfreich, wenn die Therapeut_innen als Modell fungieren können. Die eigene Erfahrung als Meditierende lässt klarer erkennen, wie herausfordernd es sein kann, auch nur kurz mit der eigenen Aufmerksamkeit beim gegenwärtigen Erleben zu bleiben, mitfühlend, ohne sich selbst $\mathrm{zu}$ bewerten oder sofort $\mathrm{zu}$ reagieren. Gerade bei komplextraumatisierten Klient_innen ist das auf eigene Erfahrung basierende Verständnis und Feingefühl wichtig, sonst laufen Therapeut_innen Gefahr, die Klient_innen unter Druck zu setzen und sie durch ihr „Versagen“ zu beschämen. Viele der oben beschriebenen Übungen klingen sehr einfach und sind doch, nicht nur für traumatisierte Menschen, eine enorm große Herausforderung, die viel Mut und Vertrauen erfordert.

Achtsamkeitstraining für Therapeut_innen kann zusätzlich Qualitäten schulen, die für die Arbeit, nicht nur mit traumatisierten Menschen, hilfreich ist. Dieses Training stärkt die wache Präsenz und die nichtbewertende Aufmerksamkeit für die innere Welt der Klient_innen (Weiss und Harrer 2010; Fulton 2009). Die Fähigkeit zur „bifokalen Wahrnehmung“ wird verfeinert, da Therapeut_innen lernen, das Erleben der Klient_innen und ihr eigenes zugleich zu beobachten. Dies erhöht die Fähigkeit zu Empathie und der Wahrnehmung von Resonanz oder Übertragungsphänomenen. So zeigt eine Studie von Grepmair und Nickel (2007), dass sich Achtsamkeitstraining günstig auf die Therapieergebnisse auswirkte, da sich die Klient_innen besser wahrgenommen fühlten.

Es ist naheliegend, dass es in diesem Arbeitsfeld sinnvoll ist, Mitgefühl und Selbstmitgefühl $\mathrm{zu}$ trainieren (Germer 2010). Auch Reddemann (2016) weist auf die besonderen Belastungen in der Arbeit mit schwer traumatisierten Menschen hin, wo es nicht immer leicht ist, offen und mitfühlend zu bleiben. Therapeut_innen spüren immer wieder die Angst vor dem Ausmaß der inneren Schmerzen traumatisierter Klient_innen, und manche verhalten sich den Therapeut_innen gegenüber auf eine Art und Weise, dass es nicht immer leicht fällt, sie zu mögen. Die Fähigkeit, immer wieder zum Mitgefühl zurückzufinden, hilft nicht nur den Klient_innen, sondern auch den Therapeut_innen, da Letztere auch in intensiven Momenten offen und erreichbar bleiben können. Das ist von großer Bedeutung, da die therapeutische Beziehung allgemein als wichtiger Wirkfaktor der Psychotherapie gilt (vgl. auch Lambert 1992).

\section{Schluss}

Ausgehend von der Definition von Achtsamkeit zeigten sich viele Gemeinsamkeiten in den Grundannahmen zur Wirksamkeit von traumatherapeutischen und achtsamkeitsbasierten Interventionen. In der Stabilisierungsphase einer Traumatherapie wird bereits eine Vielzahl von achtsamkeitsbasierten Übungen eingesetzt, und die dabei erlernten Fähigkeiten erweisen sich auch für die Konfrontationsphasen als hilfreich. Des Weiteren zeigte sich, dass der Entwicklung von Selbstmitgefühl im Heilungsprozess eine nicht $\mathrm{zu}$ unterschätzende Rolle zukommt. Eigene Erfahrungen mit dem Training von Achtsamkeit fördern bei Therapeut_innen die Fähigkeit, diese Übungen individuell abgestimmt anbieten zu können und so das Potenzial von Achtsamkeit den Klient_innen auf eine Weise zur Verfügung zu stellen, dass deren Vertrauen in die eigene Selbstwirksamkeit und in das eigene Heilungspotenzial steigen kann.

Interessenkonflikt S. Fabach gibt an, dass kein Interessenkonflikt besteht.

Open Access Dieser Artikel wird unter der Creative Commons Namensnennung 4.0 International Lizenz (http:// creativecommons.org/licenses/by/4.0/deed.de) veröffentlicht, welche die Nutzung, Vervielfältigung, Bearbeitung, Verbreitung und Wiedergabe in jeglichem Medium und Format erlaubt, sofern Sie den/die ursprünglichen Autor(en) und die Quelle ordnungsgemäß nennen, einen Link zur Creative Commons Lizenz beifügen und angeben, ob Änderungen vorgenommen wurden.

\section{Literatur}

Banks, K., Newman, E., \& Saleem, J. (2015). An overview of the research on mindfulness-based interventions for treating symptoms of Posttraumatic Stress Disorder. A systematic review. Journal of Clinical Psychology, 71(10), 935-963.

Bodhi, B. (2011). What does mindfulness really mean? A canonical perspective. Contemporary Buddhism, 12(1), 19-39. http://www.ahandfulofleaves.org/documents/Articles/ What\%20Does\%20Mindfulness\%20Really\%20Mean Bodhi_2011.pdf.Zugegriffen:30.04.2017.

Briere, J. (2014). Arbeit mit Trauma. In C. K. Germer \& R. D. Siegel (Hrsg.), Weisheit und Mitgefühl in der Psychotherapie. Achtsame Wege zur Vertiefung der therapeutischen Praxis (S. 423-446). Freiburg: Arbor. engl. Orig. 2012.

Bundschuh-Müller, K. (2009). „Es ist was es ist sagt die Liebe... "Achtsamkeit und Akzeptanz in der Personzentrierten und Experientiellen Psychotherapie. In T. Heidenreich \& J. Michalak (Hrsg.), Achtsamkeit und Akzeptanz in der Psychotherapie. Ein Handbuch (3. Aufl. S. 424-476). Tübingen:DGVT.

Ebner, F., \& Rost, C. (2008). Einleitung: Ressourcenaktivierung mitEMDR. In C. Rost (Hrsg.), Ressourcenarbeitmit EMDR. Bewährte Techniken im Überblick. Vom Überleben zum Leben (S. 7-30). Paderborn: Junfermann.

Fulton, P. R. (2009). Achtsamkeit als klinisches Training. In C. K. Germer, R. D. Siegel \& P. R. Fulton (Hrsg.), Achtsamkeit in der Psychotherapie (S. 85-110). Freiamt: Arbor. engl. Orig. 2005. 
Germer, C. K. (2009). Achtsamkeit. In C. K. Germer, R. D. Siegel \& P. R. Fulton (Hrsg.), Achtsamkeit in der Psychotherapie (S. 15-49). Freiamt:Arbor. engl. Orig. 2005.

Germer, C. K. (2010). Der achtsame Weg zur Selbstliebe. Wie man sich von destruktiven Gedanken und Gefühlen befreit. Freiburg: Arbor. engl. Orig. 2009

Grepmair, L. J., \& Nickel, M. K. (2007). Achtsamkeit des Psychotherapeuten. Wien: Springer.

Grossman, P., Niemann, L., Schmidt, S., \& Walach, H. (2009). Ergebnisse einer Metaanalyse zur Achtsamkeit als klinischer Intervention. In T. Heidenreich \& J. Michalak (Hrsg.), Achtsamkeit und Akzeptanz in der Psychotherapie. Ein Handbuch (3. Aufl. S. 729-753). Tübingen: DGVT.

Grossman, P., \& Reddemann, L. (2016). Achtsamkeit. Wahrnehmen ohne Urteilen - oder ein Weg, Ethik in der Psychotherapie zu verkörpern? Psychotherapeut, 61(3), 222-228.

Harrer, M.E., \& Weiss, H. (2016). Wirkfaktoren der Achtsamkeit. Wie sie die Psychotherapie verändern und bereichern. Stuttgart: Schattauer.

Hayes, S. C., Strosahl, K. D., \& Wilson, K. G. (2014). Akzeptanz \& Commitment Therapie: Achtsamkeitsbasierte Veränderungen in Theorie und Praxis. Paderborn: Junfermann. engl. Orig. 1999

Heidenreich, T., \& Michalak, J. (2009). Achtsamkeit und Akzeptanz in der Psychotherapie - Eine Einführung. In T. Heidenreich \& J. Michalak (Hrsg.), Achtsamkeit und Akzeptanz in der Psychotherapie. Ein Handbuch (3. Aufl. S. 11-24). Tübingen:DGVT.

Huber, M. (2005). Der innere Garten. Ein achtsamer Weg zur persönlichen Veränderung. Paderborn: Junfermann.

Huber, M. (2011). Die Affektkette. Eine Möglichkeit, Lebensthemen und Langzeitgefühle zu bearbeiten. Mülheim-Baden: auditorium netzwerk. DVD

Huppertz, M., Saurgnani, S., \& Schneider, S. (2013). Ein pluralistisches Achtsamkeitskonzept für die therapeutische Praxis. Verhaltenstherapie \& psychosoziale Praxis, 45(2), 381-397. http://www.mihuppertz.de/VPP2-13_S8. pdf.Zugegriffen:30.04.2017.

Kabat-Zinn, J. (2003). Mindfulness-based interventions in context: Past, present and future. Clinical Psychology: Science and Practice, 10(2), 144-156.

King, A. P., Erickson, T. M., Giardino, N. D., Favorite, T., Rauch, S. A. M., Robinson, E., Kulkarni, M., \& Liberzon, I. (2013). A pilot study of group mindfulness-based cognitive therapie (MBCT) for combat veterans with posttraumatic stress disorder (PTSD). Depress Anxiety, 30(7), 638-645. https:// www.ncbi.nlm.nih.gov/pmc/articles/PMC4373594/.Zugegriffen: 30.04.2017.

Lambert, M.J. (1992). Implications of outcome research for psychotherapy integration. In J.C. Norcross (Hrsg.), Handbook of psychotherapy integration (S. 94-129). New York: Basic Books.
Münker-Kramer, E. (2015). Traumazentrierte Psychotherapie mit EMDR. München: Reinhardt.

Purtscher, K., \& Sack, M. (2016). Stabilisieren oder Konfrontieren? - Aktuelles aus der Diskussion rund um Traumatherapie. Ein Interview von Silke Birgitta Gahleitner. Psychotherapie Forum, 21(4), 115-117.

Reddemann, L. (2006). Achtsamkeit in der tiefenpsychologisch fundierten Traumatherapie. Psychotherapie im Dialog, 7(3), 297-301. http://www.luise-reddemann. de/fileadmin/content/downloads/aufsaetze-vortraege/ AchtsamkeitArtikel.pdf.Zugegriffen:30.04.2017.

Reddemann, L. (2011a). Achtsamkeit in der Behandlung von persönlichkeitsgestörten und traumatisierten Patienten. In L. Reddemann (Hrsg.), Kontexte von Achtsamkeit in der Psychotherapie(S. 101-117). Stuttgart:Kohlhammer.

Reddemann, L. (2011b). Kontexte von Achtsamkeit in der Psychotherapie. In L. Reddemann (Hrsg.), Kontexte von Achtsamkeit in der Psychotherapie (S. 18-38). Stuttgart: Kohlhammer.

Reddemann, L. (2016). Mitgefühl, Trauma und Achtsamkeit in psychodynamischen Therapien. Göttingen: Vanderhoeck \& Ruprecht.

Renn, K. (2011). Focusing: Psychotherapie in innerer Achtsamkeit. In L. Reddemann (Hrsg.), Kontexte von Achtsamkeit in der Psychotherapie (S. 84-100). Stuttgart: Kohlhammer.

Roediger, E. (2011). Achtsamkeit und Schematherapie. In L. Reddemann (Hrsg.), Kontexte von Achtsamkeit in der Psychotherapie (S.9-17). Stuttgart: Kohlhammer.

Rytz, T., \& Wiesmann, S. (2016). Ankommen in der Gegenwart durch achtsame Körperwahrnehmung: Methodische Überlegungen für die Begleitung von Menschen mit traumatischenErfahrungen. Trauma - Zeitschrift für Psychotraumatologie und ihre Anwendungen, 15(2), 52-61.

Siegel, D. J. (2007). Das achtsame Gehirn (korr. Neuaufl.. Aufl.). Freiamt: Arbor.

Stephenson, K. R., Simpson, T. L., Martinez, M.E., \& Kearney, D. J. (2016). Changes in mindfulness and posttraumatic stress disorder symptoms among veterans enrolled in mindfulness-based stress reduction. Journal of Clinical Psychology, 73(3), 201-217.

Valdez, C. E., Sherrill, A. M., \& Lilly, M. M. (2016). Present moment contactand nonjudgment:Pilot data on dismantling mindful awareness in trauma-related symptomatology. Journal of Psychopathology and Behavioral Assessment, 38(4), 572-581. doi:10.1007/s10862-016-9548-8.

Weiss, H., \& Harrer, M.E. (2010). Achtsamkeit in der Psychotherapie. Verändern durch „Nicht-Verändern-Wollen" - ein Paradigmenwechsel? Psychotherapeutenjournal, 1, 14-24. http://www.mbsrkoeln.de/artikel/MBSR Veraendern.pdf.Zugegriffen:30.04.2017.

Wetzel, S. (2011). Aufmerksamkeit, Achtsamkeit und Erwachen - buddhistische Perspektiven. In L. Reddemann (Hrsg.), Kontexte von Achtsamkeit in der Psychotherapie (S.39-51). Stuttgart:Kohlhammer. 